\title{
Corrigendum to
}

\section{"Is annual recharge coefficient a valid concept in arid and semi-arid regions?" published in Hydrol. Earth Syst. Sci., 21, 5031-5042, 2017}

\author{
Yiben Cheng ${ }^{1,2}$, Hongbin Zhan ${ }^{2}$, Wenbin Yang ${ }^{1}$, Hongzhong Dang ${ }^{1}, \mathrm{Wei} \mathrm{Li}^{1}, \mathrm{Lili} \mathrm{Wu}^{1}$, and Yanli $\mathrm{Li}^{1}$ \\ ${ }^{1}$ Institute of Desertification Studies, Chinese Academy of Forestry, Haidian District, Beijing 100093, China \\ ${ }^{2}$ Department of Geology and Geophysics, Texas A\&M University, College Station, TX 77843-3115, USA \\ Correspondence to: Yiben Cheng (chengyiben07@gmail.com) and Hongbin Zhan (zhan@geos.tamu.edu)
}

Published: 16 October 2017

There are some mistakes in the article that need to be corrected.

Lili Wu and Yanli Li helped to collect data, so the first contact would like to add their names to the list of authors. Therefore, the correct author list should be Yiben Cheng, Hongbin Zhan, Wenbin Yang, Hongzhong Dang, Wei Li, Lili Wu, and Yanli Li. The affiliation and contact information of these two added authors are also Institute of Desertification Studies, Chinese Academy of Forestry, Haidian District, Beijing 100093, China. You will find the correct title page above.
The acknowledgement section needs to be corrected as well. The correct one is as follows.

Acknowledgements. This study was supported with research grants from the Natural Science Foundation of China (41771206, 41661006). 